\title{
Tradition et « agentivité » dans la rhétorique humaniste
}

Tradition and Agency in Humanistic Rhetoric

\section{Michael Leff}

Traducteur : Sivan Cohen-Wiesenfeld

\section{(2) OpenEdition}

1 Journals

Édition électronique

URL : http://journals.openedition.org/aad/1077

DOI : 10.4000/aad.1077

ISSN : 1565-8961

Éditeur

Université de Tel-Aviv

Référence électronique

Michael Leff, "Tradition et « agentivité » dans la rhétorique humaniste », Argumentation et Analyse du Discours [En ligne], 6 | 2011, mis en ligne le 15 avril 2011, consulté le 23 septembre 2019. URL : http:// journals.openedition.org/aad/1077; DOI : 10.4000/aad.1077

Ce document a été généré automatiquement le 23 septembre 2019.

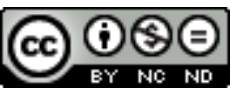

Argumentation \& analyse du discours est mis à disposition selon les termes de la licence Creative Commons Attribution - Pas d'Utilisation Commerciale - Pas de Modification 4.0 International. 


\title{
Tradition et « agentivité » dans la rhétorique humaniste
}

\author{
Tradition and Agency in Humanistic Rhetoric
}

\author{
Michael Leff
}

Traduction : Sivan Cohen-Wiesenfeld

\section{NOTE DE L'ÉDITEUR}

Cet article a paru en anglais dans Philosophy and Rhetoric 2003, 36:2, 135-147, et est publié en français avec l'autorisation de la revue.

1 J'aborderai ici la question de la tradition de manière indirecte, en prenant d'abord en considération certaines questions liées àl'agentivité rhétorique ${ }^{1}$. Cette stratégie peut sembler maladroite, voire même périlleuse, puisqu'elle enchevêtre deux concepts aussi complexes que controversés. J'espère néanmoins montrer que, dans le champ de la rhétorique, ces notions sont reliées par un lien qui influence fortement notre manière de les appréhender, bien que ce lien n'ait pas été ouvertement reconnu à ce jour. Je pose en particulier comme hypothèse que l'approche humaniste implique une conception de l'agentivité dont l'ambiguïté est féconde, et qui positionne l'orateur à la fois comme individu guidant un auditoire, et comme membre d'une communauté façonné et contraint par les exigences de l'auditoire. Cette tension s'explique, selon moi, lorsqu'on perçoit que la tradition fonctionne comme une force de médiation entre les identités individuelles et collectives. Vue sous cet angle, elle apparaît comme une ressource primordiale de l'invention rhétorique.

2 Avant d'entamer ce débat, je dois cependant expliquer dans quel sens j'utilise le terme «humaniste", puisque le concept d'humanisme, tout comme ceux de tradition et d'agentivité, est un concept doté de multiples significations qui entretiennent peu de rapports entre elles. Je voudrais donc indiquer clairement que je ne fais pas référence à une sorte de religion laïque, la religion de l'humanité, ni à la version de la philosophie pragmatique développée par William James et Ferdinand C. S. Schiller, pas plus qu'à 
l'idéologie que l'on nomme souvent «humanisme libéral». J'ai plutôt à l'esprit un courant spécifique de l'histoire de la rhétorique qui part de la Grèce antique avec Protagoras et Isocrate, reparaît à Rome sous l'égide de Cicéron et de Quintilien, puis à nouveau chez les humanistes de la Renaissance, et bénéficie jusqu'à ce jour de l'attention et $\mathrm{du}$ respect de certains rhétoriciens contemporains ${ }^{2}$. La personnalité dominante dans l'histoire de ce courant étant Cicéron, on le qualifie souvent d'humanisme cicéronien. Bien que ses manifestations varient considérablement suivant les lieux et les époques, certaines de ses caractéristiques restent néanmoins constantes. Elles comprennent une certaine méfiance à l'égard de la théorie abstraite non seulement dans le domaine de la rhétorique, mais aussi dans celui de l'éthique et du politique $^{3}$; la conviction que le discours, particulièrement celui qui permet le débat contradictoire, joue un rôle fondamental dans la vie publique ${ }^{4}$; une valorisation et une idéalisation de l'éloquence qui implique un lien étroit avec la vertu ${ }^{5}$; et une conception de celle-ci qui la relie résolument à l'activité politique ${ }^{6}$. Dans les développements qui suivent, j'espère montrer que certains points de vue sur l'agentivitéet la tradition relèvent également de cet ensemble d'attitudes communes.

\section{L'agentivité dans la rhétorique humaniste}

3 L'un des jugements les plus largement répandus sur la rhétorique humaniste traditionnelle parmi les chercheurs contemporains en rhétorique est qu'elle met fortement, voire exclusivement, l'accent sur la personne du rhéteur. Robert Scott résume cette position en une phrase: "Il est traditionnel de considérer le locuteur comme actif et l'auditoire comme passif» (Scott 1975: 440), et Wayne Brockreide développe ce point en affirmant que la perspective qui place «le locuteur au centre de la transaction [rhétorique] » est

répandue tout au long de l'histoire. Des sophistes et des écrits d'Isocrate et de Cicéron jusqu'à la floraison des manuels sur la prise de parole en public au vingtième siècle, la transaction rhétorique a été considérée comme étant le fait d'un locuteur qui cherche à imposer ses vues à l'auditoire pour obtenir à la fois un résultat immédiat, et le pouvoir ou la gloire en tant que membre respecté de la société (1971 : 124-125)'.

La remarque de Brockreide repose sur un soubassement politique et éthique explicité par Lois McNamara Byham: "l'ancienne rhétorique était unidirectionnelle et dotait ainsi ceux qui jouissaient de l'autorité du pouvoir de transmettre l'information à leurs subalternes ». Son but, ajoute-t-elle, «était de manipuler et d'exploiter» (1979: 22). Plus récemment, Dilip Gaonkar a traduit ces observations dans un jargon post-moderne lorsqu'il a identifié la rhétorique classique avec une «idéologie de l'agir humain » qui, entre autres, considère le locuteur comme un " lieu d'origine plutôt que comme un point d'articulation » $(1997: 32)^{8}$.

5 Les traités de rhétorique classique contiennent de nombreux éléments allant dans le sens des arguments que je viens de citer. Le dispositif technique est alimenté et organisé du point de vue du locuteur ; les rhétoriciens humanistes présentent l'orateur comme un héros culturel et célèbrent la grandeur et l'autonomie apparente du pouvoir oratoire. L'un des exemples les plus anciens et les plus remarquables de cet hommage au rhéteur apparaît dans le Gorgias de Platon. Dans ce dialogue, Gorgias affirme sans modestie aucune que les rhétoriciens dominent complètement le débat public. Ils peuvent persuader mieux qu'un spécialiste de n'importe quel sujet donné, et écraser 
«toute opposition quelle qu'elle soit », personne ne peut s'adresser à un public " avec une plus grande force de persuasion [...] Ah, si grande est la puissance de cet art rhétorique ! » (456b).

6 Platon, bien sûr, n'est pas un ami de la rhétorique, et il ne présente ce point de vue que pour mieux le réfuter. Mais le témoignage des rhétoriciens eux-mêmes prouve que son personnage de rhétoricien n'était pas un homme de paille. Isocrate, dans son Antidosis, propose un hymne élégant au pouvoir du discours qui associe l'éloquence rhétorique à une pensée saine, et conclut en affirmant que ce pouvoir est si important «que rien de sage ne se fait sans le discours, que le discours est le guide de toute pensée ou action, et que les personnes les plus intelligentes l'utilisent le plus souvent possible » (257) ${ }^{9}$. Ce pouvoir appartient au locuteur, et Isocrate explique ailleurs que la maitrise de l'éloquence requiert beaucoup de travail et est «l'œuvre d'un esprit courageux et pénétrant" (Contre les sophistes, 17). Cicéron traite des mêmes thèmes. Dans De l'invention oratoire (De inventione), l'orateur apparaît comme l'agent héroïque qui, en combinant sagesse et éloquence, persuade l'humanité d'abandonner sa condition naturellement sauvage pour adopter un mode de vie plus juste et civilisé (voir 1.2.3). Dans De l'Orateur (De oratore), Cicéron remodèle ce topos pour réunir les caractéristiques de l'éloquence dont il fait l'éloge :

Certainement rien [...] ne me semble plus beau que de pouvoir, par la parole, retenir l'attention des hommes assemblés, séduire les intelligences, entraîner les volontés à son gré, en tous sens. [...] Oui ! qu'y a-t-il de plus admirable que de voir, en face d'une immense multitude, un homme se dresser seul et, armé de cette faculté que chacun a cependant reçu de la nature, en user comme il est seul alors, ou presque seul, en mesure de le faire ? [...] Quelle puissance que celle qui dompte les passions $\mathrm{du}$ peuple, triomphe des scrupules des juges, ébranle la fermeté du Sénat, merveilleux effet de la voix d'un seul homme ? Est-il aussi rien de plus royal, peuton dire, de plus grand et généreux que de secourir les suppliants, de relever les malheureux à terre, d'arracher ses concitoyens à la mort, aux dangers, à l'exil ? (1993 : VIII. 31-32).

7 Ces sentiments cicéroniens résonnent à travers l'histoire de l'humanisme. Lors du renouveau des études classiques au douzième siècle, par exemple, John de Salisbury a défini l'orateur éloquent comme celui qui exprime convenablement et efficacement son intention ", et il a lui-même quelque mal à "concevoir comment quelque chose pourrait être globalement plus profitable; plus utile pour acquérir la richesse, plus fiable pour gagner des faveurs, plus adapté pour obtenir une réputation, que ne l'est l'éloquence » (1962: 26). Deux siècles plus tard, l'humaniste florentin Coluccio Saltuti offre une autre variation sur ce thème lorsqu'il affirme que rien n'est
plus important que de contrôler les mouvements de l'esprit, de conduire son auditeur où on le souhaite et de le ramener à nouveau là où il se trouvait, plaisamment et avec amour. Tels sont, si je ne me trompe, les pouvoirs de l'éloquence; c'est là son ouvrage. Toute la force et le pouvoir des rhétoriciens tendent à cela. C'est, bien sûr, une grande chose que d'orner l'écriture avec des mots et des maximes, mais la plus grande de toutes - sans doute embellie et dignifiée par le langage - est d'émouvoir l'esprit des auditeurs. Seule l'éloquence peut obtenir ces résultats (cité dans Seigel 1968 : 76-77).

Cette liste pourrait être considérablement étendue, mais ce court échantillon suffit à justifier la conclusion que la rhétorique humaniste valorise l'agent individuel et se concentre sur lui. Il existe néanmoins un autre aspect de la question, qui vient compliquer cette conception apparemment simple del'agentivité. John Witherspoon fait allusion à cet «envers » del'agentivitérhétorique lorsqu'il observe que pour 
atteindre son plus haut niveau, cet art de la parole doit coïncider avec « la plus grande réserve et abnégation dans son utilisation, sans quoi elle manquerait son propre but " (1990 : 232). C'est-à-dire que le pouvoir de l'orateur implique ironiquement l'humilité devant son auditoire, parce que le pouvoir d'émouvoir et de persuader celui-ci exige de s'ajuster et de s'adapter à ses sentiments. L'auditoire contraint nécessairement les horizons intellectuels de l'orateur, ses modes d'expression et jusqu'à la représentation de soi ; dès lors, si les orateurs veulent exercer une influence, ils doivent le céder aux personnes qu'ils cherchent à influencer.

Isocrate et Cicéron notent et soulignent cette dépendance de l'orateur par rapport à l'auditoire et au contexte social. C'est pourquoi Isocrate, bien qu'il souligne parfois l'imagination hardie nécessaire à la réussite d'un discours politique, prévient également son lecteur qu'il ne doit pas s'attendre à trouver chez lui des innovations, puisque qu'il y va d'un type de discours qui ne laisse « pas de place au paradoxe, ni à ce qui est invraisemblable ou non conventionnel. Mieux vaut considérer que l'orateur le plus accompli est celui qui est capable d'assembler le plus d'idées acceptées par les autres et de les articuler le plus élégamment possible » (A Nicocles, 44). Aussi, Cicéron (1985 : 12, 1.12) explique-t-il que :

L'art de la parole [...] est à découvert, à la portée, pour ainsi dire, et à la disposition de chacun, instrument d'une pratique journalière, langage usuel de la conversation; si bien que dans les autres genres on excelle à proportion qu'on s'écarte davantage de l'intelligence et de la compréhension du vulgaire; mais dans l'éloquence, ce serait la plus grave des fautes que de rejeter les façons de s'exprimer, les façons de penser et de sentir communes à tous les hommes .

Les orateurs doivent adapter leur façon de penser au « sentiment du vulgaire » et régler leur éloquence sur le "goût du grand nombre »; pour plaire, ils doivent étudier les dispositions de leurs auditeurs et se plier à leur jugement, à leur fantaisie (XXIII. 108).

11 Dans le premier livre de De oratore, Cicéron illustre cette question de manière dramatique. Lorsque Crassus, l'un des deux protagonistes du dialogue, aborde la question de la lourde charge qui pèse sur l'orateur, il insiste sur l'anxiété que provoquent en lui les exigences de son auditoire. Sa propre expérience lui a appris que les meilleurs orateurs sont ceux qui sont le plus effrayés par "les difficultés de l'art ", « l'incertitude du succès » et « l'attente des auditeurs » (1993:XXV. 118) Il confesse que lui-même " pâlit » et « tremble de tous ses membres » (ibid.) lorsqu'il prend la parole, et il rapporte une anecdote dans laquelle il fut lui-même tellement dominé par la peur que le magistrat qui présidait la cour dût suspendre le procès. Il faut se rappeler que Crassus est décrit comme un personnage imposant. Les autres protagonistes du dialogue affichent envers lui une attitude qui va du profond respect à la vénération ; c'est un dirigeant politique remarquable et un éminent orateur, dont le talent particulier est de faire naître l'émotion. Paradoxalement, donc, cet homme qui sème la crainte dans le cœur de ses auditeurs est lui-même empli de crainte devant eux.

12 Comme Jerrold Seigel l'a montré, les humanistes de la Renaissance, en grande partie en raison de leur allégeance aux notions chrétiennes de vertu, furent forcés de reconnaître le paradoxe implicite contenu dans cette perspective dualiste sur l'orateur et ne ménagèrent pas leurs efforts pour le résoudre (Seigel 1968). Chez les auteurs classiques, cependant, aucune tension de ce genre n'apparaît ; les deux perspectives coexistent à l'intérieur d'un modèle symétriquement équilibré: l'orateur guidait le peuple, infléchissait sa volonté, et obtenait ses applaudissements, mais il était 
également guidé par le peuple, soumis à sa volonté et dépendant de ses applaudissements. L'orateur est simultanément actif et passif.

13 Les critiques de l'ars oratoria (et les humanistes qui lui sont associés) ne reconnaissent pas cette complexité et considèrent que l'agentivitéest forcément l'un ou l'autre. Comme l'indique le commentaire de Gaonkar, la tendance post-moderne est d'associer la rhétorique humaniste à une conception de l'orateur comme agent autonome. Par contre, les penseurs des Lumières condamnèrent l'ars oratoria pour la raison opposée. Dans la mesure où il exige de s'adapter aux circonstances concrètes, aux mobiles et aux intérêts de l'auditoire, il rend l'orateur hétéronome et donc incapable d'exercer une liberté créative, ou d'exercer sa raison clairement et sans entraves. Locke, dans un passage maintenant couramment cité, affirme que les dispositifs rhétoriques n'ont d'autres buts que « d'insinuer de mauvaises idées, de remuer les Passions, et d'induire par là le jugement en erreur, et ne sont donc que tricherie » (1979: 508). Kant considère l'art rhétorique de la persuasion comme suspect parce qu'il trompe par un beau spectacle et satisfait la faiblesse humaine pour promouvoir des « desseins personnels ». Il n'importe pas, pour Kant, que de tels desseins fussent «bien intentionnés ou véritablement sincères ", puisqu'un art basé sur cette contrainte d'adaptation n'est, par principe, digne d'aucun respect (1966:172, n. 50).

14 L'antipathie des Lumières envers la rhétorique humaniste provient de nombreuses causes, dont l'une des principales (qui a une influence toute particulière sur la question de l'agent) est le discrédit systématique jeté sur la tradition. Selon Alisdair MacIntyre, la tradition s'est écroulée sous le poids de la rationalité des Lumières parce qu'elle ne commence jamais par "des vérités évidentes et inattaquables", et qu'elle ne peut jamais se donner pour objectif final une rationalité universelle. Les traditions sont toujours ancrées dans les contingences de l'histoire, toujours sujettes aux changements internes, et «toujours ancrées de façon indéracinable dans le local» (1988: 361). Appliqué à la question de l'agent, ceci signifie que quiconque pense ou agit en termes de tradition ne peut passer l'épreuve du jugement autonome requis par l'épistémologie des Lumières.

15 La tradition implique une conception de l'agent et du moi différente de celle de la modernité. Au lieu d'un moi isolé (ou aliéné, dans certaines versions post-modernes), la tradition construit le moi à travers l'interaction sociale et comme partie d'une évolution historique continue. Tant l'agent individuel que la tradition réalisent et transforment l'identité à travers une circulation réciproque d'influences. L'intégration dans une tradition façonne le moi individuel, mais en revanche, l'individu soumis aux mœurs et pratiques de la communauté gagne aussi le pouvoir de façonner la tradition. De plus, les agents qui parviennent à remanier la tradition transforment également la conception de leur moi puisque l'individu et les identités affiliatives ne sont jamais coupés l'un de l'autre. Ralph Ellison explique comment fonctionne ce processus dans la tradition musicale du jazz: "Chaque moment authentique de jazz jaillit d'une lutte dans laquelle l'artiste défie tout le reste ; chaque solo ou improvisation représente (comme les toiles successives d'un peintre) une définition de son identité : en tant qu'individu, comme membre de la collectivité, et comme maillon dans la chaîne des traditions » (cité dans Bone 1999: 6). La fluidité de cette relation entre l'individu et la communauté semble aussi correspondre à la conception de l'agentivité qui ressort implicitement de la rhétorique humaniste. A l'instar du musicien de jazz, l'orateur guide la communauté en fusionnant avec elle, et définit son moi non comme une 
essence immobile et isolée, mais quelque chose qui se réalise dans et par la performance publique. L'individu et le collectif, comme l'a dit Greg Clark, « interagissent dans des relations de collaboration » $(2003)^{10}$.

\section{Tradition et performance rhétorique : le cas d'Isocrate}

16 J'ai jusqu'à présent essayé de montrer que la conception humaniste de l'agentivitérhétorique implique une interaction complexe entre l'orateur et l'auditoire, et qu'elle acquiert son sens quand on reconnaît la valeur de la tradition comme médiation entre l'individu et la communauté. Cette affirmation présuppose l'existence d'une relation intime et privilégiée entre la rhétorique humaniste et la tradition : c'est ce point que j'entends à présent expliciter et justifier. Ma thèse est que pour les rhétoriciens humanistes, la tradition est la source et le fondement du discours civique, puisque celui-ci repose sur l'identité de la communauté et œuvre à sa consolidation, en même temps qu'elle fonctionne comme instrument de changement. Dans une perspective humaniste, la rhétorique possède une force constitutive parce qu'elle se situe à l'intersection de la mémoire passée et des préoccupations du présent, et permet de les équilibrer et de les ajuster. C'est là, sans doute, une affirmation très générale et ambitieuse, et je n'ai pas la prétention de la traiter intégralement dans ce court aperçu. Mais j'espère en montrer la vraisemblance en me référant à Isocrate, cas original qui remonte aux origines de la rhétorique humaniste.

Longtemps considéré comme un penseur quelconque et peu original, Isocrate fait à présent l'objet d'un intérêt renouvelé. Les recherches récentes à ce sujet, impressionnantes à la fois par leur quantité et par leur qualité, ouvrent de nouvelles perspectives sur son programme d'éducation, sa place dans l'Athènes du quatrième siècle av. J.-C., et l'importance de sa contribution à la pensée humaniste ${ }^{11}$. Trois aspects de ce renouveau sont particulièrement intéressants pour notre propos : (1) A l'inverse de l'opinion traditionnellement établie, Isocrate et Platon ne doivent pas être situés aux antipodes l'un de l'autre, l'un représentant le réalisme pratique et l'autre l'idéalisme. En effet, si Isocrate rejette vigoureusement la croyance de Platon en une vérité abstraite et objective, il est également opposé à l'extrême relativisme de Gorgias et des autres sophistes. Isocrate tente de se positionner entre Platon et Gorgias en construisant un ensemble de normes pratiques de la connaissance qui réponde à l'expérience ordinaire, se développe par le moyen du discours politique, et qui permette de façon générale d'émettre un jugement approprié sur des cas particuliers. (2) Comme Platon, Isocrate se préoccupe beaucoup de l'éducation à la vertu et sa version de la paideia, bien que plus axée sur la pratique que celle de Platon, contient une bonne part d'idéalisme. Pour Isocrate, le but de l'éducation rhétorique est l'excellence du discours délibératif, qui ne peut se réduire au simple fait de plaire à l'auditoire ou de le persuader. Elle exige au contraire de l'auto-discipline, une vaste étendue de connaissances et un sens profond du bien commun, et doit donc refléter et manifester des vertus intimement liées au caractère moral. De plus, dans la mesure où ni la capacité technique ni l'efficacité persuasive ne déterminent l'excellence du discours délibératif, il s'agit d'un concept idéalisé. C'est-à-dire qu'il doit se référer à des normes qui transcendent le contexte empirique immédiat et reposent sur des principes de vertu raisonnablement stables et durables. (3) Compte tenu de la théorie pragmatique de la connaissance d'Isocrate et de sa conception entièrement sociale et 
politique de la vertu, ces principes ne pouvaient provenir d'une source purement abstraite. Ils devaient venir de l'expérience de la communauté et, par conséquent, les idéaux d'Isocrate ne pouvaient dériver que de l'histoire ou, plus spécifiquement, de la tradition politique athénienne. En résumé, la rhétorique isocratienne est intrinsèquement ancrée dans la tradition ${ }^{12}$.

Fidèle à l'aversion humaniste pour la théorie, Isocrate n'a jamais écrit de traité conceptuel systématique de sa méthode. Il a préféré enseigner par l'exemple, et a écrit des discours politiques engagés dans des situations particulières, même lorsqu'ils illustrent la manière dont un rhéteur peut et doit mobiliser la tradition. L'un de ces exemples les plus directs et les plus intéressants est l'Areopagitica, discours qui a fait l'objet d'une étude approfondie de la part de deux des principaux défenseurs de la l'approche réactualisée d'Isocrate, Josiah Ober et David Konstan ${ }^{13}$. Dans ce qui suit, je ferai des emprunts importants à ces deux auteurs pour expliquer comment l' Areopagitica à la fois se fonde sur la tradition et l'utilise dans des buts rhétoriques.

19 Ce discours fait partie de l'effort général d'Isocrate pour réformer la politique athénienne dans une direction conservatrice. Son but était de persuader ses concitoyens de réviser la forme populiste de démocratie existante pour la remplacer par une constitution plus restreinte - une variante du gouvernement démocratique dans laquelle les masses s'en remettraient volontairement à des dirigeants d'élite qui, aux yeux d'Isocrate, posséderaient les dispositions nécessaires pour exercer un jugement politique judicieux. Le discours est extrêmement critique à l'égard de la démocratie athénienne telle qu'elle était pratiquée au milieu du IVe siècle av. J.-C., mais il évite soigneusement, et habilement, de critiquer le gouvernement démocratique en lui-même. La rhétorique du texte implique donc une dissociation entre le régime populiste actuel et un autre type de démocratie athénienne, meilleur, identifié à l'ancien régime fondé par Solon et rétabli par Clisthène. Isocrate célèbre la constitution de Solon à la fois en raison des vertus qu'elle incarne et stimule et pour les avantages matériels qu'elle apporta à Athènes. Par contraste, il présente la démocratie populiste existante comme péchant par manque de modération, enclin à confondre la licence et la liberté, et à la source de la ruine matérielle du pays à l'intérieur comme à l'extérieur.

Comme l'a démontré Konstan, ce discours repose sur l'autorité de Solon, puisqu'Isocrate présente la constitution solonienne comme l'authentique source de la tradition démocratique athénienne et comme le modèle d'une version réformée et restreinte de gouvernement démocratique appropriée à sa propre époque. L'autorité de Solon, bien sûr, n'est pas une donnée historique inerte. Elle n'est pas inscrite dans une histoire « objective » d'Athènes, et ne fait pas encore partie de la mémoire collective des citoyens athéniens du quatrième siècle. C'est une possibilité rhétorique qu'Isocrate peut exploiter à ses propres fins. Isocrate choisit Solon comme pierre de touche de la tradition athénienne, et non Thésée ou quelque autre personnage dont le choix était également envisageable, car cette alternative correspond à ses intérêts politiques immédiats ; il explique la nature et les conséquences du régime solonien en des termes qui font pencher l'histoire de façon décisive en faveur de son attitude envers le présent. La tradition n'est pas infiniment malléable, si bien qu'Isocrate ne peut tout se permettre ; il ne peut pas raconter des histoires sans fondement aucun dans la mémoire culturelle athénienne, ni présenter les personnes et les évènements d'une manière qui offense la sensibilité de son auditoire. Ces limites lui laissent cependant un espace 
suffisant pour effectuer son travail rhétorique et fonder ses objections au régime existant sur un contexte historique plausible.

Lu dans cette perspective, l'Areopagitica devient un exercice d'utilisation rhétorique et herméneutique de l'histoire. La tradition athénienne sert de cadre au raisonnement et le justifie, mais cette tradition est fabriquée (ou du moins modelée) dans le texte, qui travaille à construire une interaction réciproque entre le passé et le présent. Dans la mesure où elle possède un but persuasif, la représentation que donne Isocrate de la constitution solonienne transforme en même temps la compréhension de son référent historique que celle de son contexte politique immédiat. La tradition cerne le champ rhétorique et le contraint, mais elle reste aussi ouverte aux nouvelles constructions rhétoriques, de sorte qu'elle est capable de «se développer tout en maintenant son identité et sa continuité » (Pelikan $1984: 58$ ).

Avant de conclure, nous devons également faire référence à la lecture de l'Areopagitica de Josiah Ober, quelque peu différente mais néanmoins complémentaire de la nôtre. Evoquant la conception de la critique immanente chez Michael Walzer, Ober souligne les différences entre Platon et Isocrate sur le plan politique en mettant l'accent sur certaines caractéristiques distinctives de la paideia isocratienne. Il note qu'à l'instar de Platon, Isocrate considérait la démocratie populiste avec mépris mais que, par contre, il n'aurait pas complètement abandonné le processus politique pour adopter une perspective étrangère aux pratiques et aux attitudes en cours. Isocrate ne rejetait donc pas la démocratie en faveur d'un type de gouvernement plus cohérent sur le plan abstrait ; il établissait plutôt des distinctions entre les diverses formes de démocratie et a repéré (ou inventé) des alternatives à partir de l'histoire du régime politique :

Plutôt que de travailler tantôt en s'écartant et tantôt en se rapprochant de la connaissance fondamentale de la réalité, plutôt que d'essayer de fonder la politique sur une appréhension solide du monde tel qu'il est réellement, Isocrate reste enraciné dans sa société. Isocrate se représente comme [...] un critique de l'intérieur, qui considère que son rôle est d'appeler ses concitoyens à rester fidèle aux idéaux les plus élevés de leur propre tradition politique $(2000: 282)$.

23 J'ajouterais que cette position immanente semble presque inévitablement coïncider avec l'humanisme d'Isocrate. Dans la mesure où, selon sa version de la Paideia, il considère la vertu comme un accomplissement social et comme un élément disséminé dans le discours politique et régulé par lui, Isocrate ne peut pas sortir du politique. Par conséquent, le champ de sa pensée est tributaire des forces sociales et politiques existantes; mais celles-ci contribuent également à sa virtuosité rhétorique car elles offrent des ressources à des modes d'expression novateurs et des formes d'argument qui fonctionnent dans le cadre de la tradition tout en travaillant à son évolution.

La conception selon laquelle la tradition facilite l'inventivité peut sembler étrange à ceux d'entre nous qui, vivant dans le sillage de la rationalité des Lumières, ont été habitués à penser que "la tradition est comme une livre de thé, qui nous permet de brasser exactement la même boisson que nos pères " (McCaughey 1997 : 56), ou, pour poser le problème de manière plus abstraite à la manière de Lyotard et Thébaud, que la tradition est « un rapport au temps qui serait un rapport de conservation, où ce qui serait important, ce serait de garder les choses pour les préserver d'une usure temporelle » $(1979: 66)$. Comme le montre le cas d'Isocrate, cependant, les rhétoriciens humanistes présentent et développent une conception beaucoup plus vivante de la tradition, qu'ils conçoivent comme une force vivante nécessitant une évolution et une adaptation constantes pour remplir sa fonction stabilisante. Sans le support conceptuel 
qu'elle lui fournit, l'art rhétorique ne pourrait pas exister, car l'orateur ne disposerait pas d'une plateforme suffisamment stable pour réaliser sa performance. Mais cette base n'est stable que temporairement et dans un lieu déterminé, car elle est elle-même constamment modifiée par les performances rhétoriques qu'elle rend possible. L'art rhétorique est donc tributaire de la tradition qui lui sert de base et limite le champ de sa performance, mais c'est aussi la tradition qui fait de la performance rhétorique une partie significative de l'histoire de la communauté et lui donne la possibilité de se renouveler sans perdre le sens de son identité.

Pour finir, et pour revenir à mon thème de départ, cette conception de la tradition semble intimement liée à une approche ambiguë et interactive de l'agentivitérhétorique. Puisque la tradition ne peut être préservée dans sa forme première et originale, elle doit être vivifiée par des interventions qui interprètent la relation entre le passé et le présent. Pour les rhétoriciens humanistes, les agents de cette intervention sont les orateurs. Pour réussir, ils doivent persuader la communauté de modifier son attitude envers l'histoire, et cette tâche implique un effort de collaboration dans lequel la mémoire commune peut se dilater, se recentrer, et se transformer selon ce qu'exigent les circonstances. L'orateur doit donc faire preuve d'une virtuosité individuelle en présentant et en représentant la tradition tout en affirmant sa solidarité avec l'auditoire. Les orateurs usent d'un medium à la fois limité et souple; ils doivent faire preuve d'une grande créativité pour le manipuler sans paraître le subvertir, exercice qui exige d'établir un rapport délicat et sensible au contexte entre le moi et la communauté - faculté qui permet à certains individus de se distinguer et de se poser en dirigeants parce qu'ils se tiennent aux côtés et au service de ceux qu'ils dirigent.

\section{BIBLIOGRAPHIE}

Atwill, Janet. 1993. «Instituting the Art of Rhetoric : Theory, Practice, and Productive Knowledge in Interpretation ", Rethinking the History of Rhetoric : Multidisciplinary Essays on the Rhetorical Tradition, Poulakos, Takis (éd.). (Boulder : Westview Press), 91-118

Bone, Robert. 1999. «Ralph Ellison and the Uses of Imagination », Modern Critical Interpretations : Invisible Man, Bloom, Harold (éd.). (Philadelphia : Chelsea House Publishers), 3-14

Brockreide, Wayne. 1971. «Trends in Rhetoric : Blending Criticism and Science ", The Prospect of Rhetoric, Bitzer, Lloyd \& Edwin Black (eds) (Englewood Cliff, NJ : Prentice Hall), 124-125

Byham, Lois McNamara. 1979. «Rhetoric as Epistemic : A Reexamination », Rhetoric 78, Brown, Robert \& Martin Steinman (eds) (Minneapolis : University of Minnesota Center for Advanced Studies in Language, Style, and Literary Theory), 22-33

Campbell, John Angus. 1997. « Strategic Reading : Rhetoric, Intention, and Interpretation », Gross, Alan \& William Keith (eds). Rhetorical Hermeneutics : Invention and Interpretation in the Age of Science, (Albany : SUNY P), 113-37 
Charland, Maurice. 2003. « The Constitution of Rhetoric's Tradition » Philosophy and Rhetoric 36-2, 119-134

Cicéron. 1939. Orator and Brutus (Cambridge, MA : Harvard UP), trans. H. M. Hubbell

Cicéron. 1985. De l'orateur (De oratore), texte établi et traduit par Edmond Courbaud, (Paris : Les Belles Lettres)

Clark, Gregory. 2004. « Virtuosos and Ensembles : Rhetorical Lessons from Jazz »,Kent, Thomas \& Barbara Couture (eds). The Private, The Public, and the Published: Reconciling Private Lives and Public Rhetoric (Logan : Utah State University Press), 31-46

Conley, Thomas. 1990. Rhetoric in the Western Tradition (New York : Longman)

Eden, Kathy. 1997. Hermeneutics and the Rhetorical Tradition : Chapters in the Ancient Legacy and Its Humanist Reception (New Haven : Yale UP.)

Fleming, David. 1998. « Rhetoric as a Course of Study », College English 61, 91-169

Gaonkar, Dilip. 1997. «The Idea of Rhetoric in the Rhetoric of Science », Gross, Alan \& William Keith. Rhetorical Hermeneutics: Invention and Interpretation in the Age of Science (Albany : SUNY P. ), $25-88$

Isocrate. 1843. Contre les sophists, trad. d'Aimé-Marie-Gaspard de Clermont-Tonnerre (http:// remacle.org/bloodwolf/orateurs/isocrate/sophistes.htm)

John of Salisbury. 1962. The Metalogicon of John of Salisbury: A Twelfth-Century Defense of the Verbal and Logical Arts of the Trivium,trans. David D. McGarry (Berkeley and Los Angeles : University of California Press)

Jost, Walter. 1991. « Teaching the Topics : Character, Rhetoric, and Liberal Education », Rhetoric Society Quarterly 21, 1-16

Kahn, Victoria. 1997. « Humanism and the Resistance to Theory », Walter, Jost \& Michael Hyde (eds). Rhetoric and Hermeneutics in Our Time: A Reader (New Haven : Yale UP), 149.70

Kant, Immanuel. 1966. Critique of Judgment (New York : Hafner), trans. J. H. Bernard

Lanham, Richard. 1993. The Electronic Word: Democracy, Technology and the Arts (Chicago :

University of Chicago Press )

Lanham, Richard. 1976. The Motives of Eloquence : Literary Rhetoric in the Renaissance (New Haven : Yale UP)

Locke, John. 1979. An Essay on Human Understanding (Oxford : Oxford UP)

Lyotard, Jean-Francois, \& Jean Loup Thébaud. 1979. Au juste. Conversations (Paris : Christian Bourgois)

MacIntyre, Alistair. 1988. Whose Justice? Which Rationality? (Notre Dame : University of Notre Dame Press )

McCaughey, Davis. 1997. Tradition and Dissent (Melbourne : Melbourne UP)

Mendelson, Michael. 1997. «Everything Must Be Argued : Rhetorical Theory and Pedagogical Practice in Cicero's De orator ", Journal of Education 179, 15-47

Mendelson, Michael. 2002. Many Sides : A Protagorean Approach to the Theory, Practice and Pedagogy of Argument (Dordrecht : Kluwer) 
Ober, Josiah. 1999. «Eloquence, Leadership, Memory : Isocrates Antidosis and Areopagiticus », Political Dissent in Democratic Athens : Intellectual Critics of Popular Rule (Princeton : Princeton University Press), 89-248

Platon. 1993. Gorgias, présentation et trad. Monique Canto (Paris : Garnier-Flammarion)

Pelikan, Jaroslav. 1984. The Vindication of Tradition (Yale : Yale University Press)

Poulakos, Takis. 1997. Speaking for the Polis : Isocrates. Rhetorical Education (Columbia : University of South Carolina Press )

Poulakos, Takis, \& David Depew (eds). 2004. Isocrates and Civic Education (Austin : University of Texas Press )

Scott, Robert. 1975. «A Synoptic View of Systems of Western Rhetoric», Quarterly Journal of Speech $61,47-440$

Seigel, Jerrold E. 1968. Rhetoric and Philosophy in Renaissance Humanism : The Union of Eloquence and Wisdom from Petrarch to Valla (Princeton : Princeton University Press)

Sloane, Thomas. 1997. On the Contrary: The Protocol of Traditional Rhetoric (Washington, D.C. : Catholic University of America Press)

Stuerver, Nancy. 1970. The Language of History in the Renaissance: Rhetoric and Historical Consciousness in Florentine Humanism (Princeton : Princeton University Press)

Too, Yun Lee. 1995. The Rhetoric of Identity in Isocrates : Text, Power, Pedagogy (Cambridge : Cambridge UP)

Witherspoon, John. 1990. Selected Writings of John Witherspoon. Thomas Miller (ed.) (Carbondale : Southern Illinois UP. )

\section{NOTES}

1. Le terme d'agentivité est généralement retenu en français pour rendre " agency ", une notion couramment utilisée dans le domaine de la rhétorique anglo-saxonne, et qui se rapporte globalement à la capacité qu'a l'orateur d'agir par sa parole de façon délibérée et programmée. Cf. l'introduction, dans ce numéro, de R. Amossy (Note de la rédaction).

2. Parmi ceux-ci, on peut citer Fleming (1998), Jost (1991), Lanham (1976 et 1993), Mendelson (1997 et 2002), et Sloane (1997). Ce n'est peut-être pas une coïncidence si chacun de ces auteurs s'avère être soit (a) un spécialiste de la Renaissance, soit (b) particulièrement intéressé par le lien entre l'histoire de la rhétorique et la pédagogie, soit (c) les deux à la fois.

3. Voir Eden (1997) et Kahn (1997).

4. Voir Mendelson (1997) et Sloane (1997).

5. Voir Lanham (1993 : 186-192).

6. Pour un regard plus en détail sur la rhétorique humaniste, voir Conley (1990, en part. 17-20, 34-42, et 109-150) et Stuerver (1970: 5-39).

7. Il est intéressant de noter que Brockreide considère Aristote comme une exception possible ou partielle à cette concentration aveugle sur l'agent rhétorique. Il s'agit, je pense, d'une indication involontaire mais très claire, qui révèle à quel point la tradition humaniste (représentée ici par Isocrate et Cicéron plutôt que par Aristote) est associée aux intérêts manipulatoires de l'orateur, Elle montre aussi la confusion provoquée par les chercheurs contemporains qui ne parviennent pas à distinguer la rhétorique à orientation théorique de celle tournée vers la pratique et l'enseignement. 
8. L'évolution de la ligne orthodoxe du modernisme de Brockreide au post-modernisme de Gaonkar s'est heurtée à certaines résistances. Lanham, par exemple, présente une vue beaucoup plus fluctuante de l'agentivité dans la tradition (voir en part. 1993 : 186.91), bien qu'il ne se préoccupe pas directement ni prioritairement de cette question. Atwill (1993) offre une conception de la "connaissance productive" ancrée dans la tradition qui suggère une conception de l'agentivité différente de celle de la version standard, et plus ambiguë ; mais elle ne se concentre pas non plus directement sur la question de l'agentivité. John Angus Campbell, en revanche, dans une vigoureuse réponse à Gaonkar, traite explicitement et en détail de l'agentivité, mais ses arguments ne se réfèrent directement ni à la tradition ni à l'interprétation de Gaonkar ou des premiers partisans d'une conception dominée par l'agent (voir Campbell 1997 : 113-137, en part. 118-123).

9. Notre traduction.

10. Une relation similaire entre l'individu et la collectivité a été observée chez les conteurs des sociétés traditionnelles. Jean-Francois Lyotard et Jean Loup Thébaud notent que : le sujet de l'énonciation n'a aucune revendication d'autonomie quant à son discours. Au contraire, à la fois par son nom [storyteller = raconteur d'histoires] et par l'histoire qu'il raconte, il réclame son appartenance à la tradition [...] La caractéristique pertinente [d'une bonne histoire] n'est pas la fidélité [...] A l'inverse, c'est parce qu'il exagère, qu'il invente de nouveaux épisodes qui se détachent comme des motifs sur la toile de fond de l'intrigue narrative, qui, elle, reste stable, que le conteur a du succès (Lyotard et Thebaud 1979 : 33). Maurice Charland, dont l'article dans ce numéro de Philosophy and Rhetoric développe ce thème plus en détail, à attiré mon attention sur ce passage.

11. Parmi les exemples les plus importants de cette littérature, on peut citer Ober (1999), Poulakos (1997), Poulakos \& Depew (2004) et Too (1995).

12. Cette idée est développée plus en détail dans mon article «Isocrates, Tradition, and the Rhetorical Version of Civic Education », dans Poulakos \& Depew (2004).

13. Ober, $(1999,89-277)$ et l'article de Konstan «Isocrates' Republic » dans Poulakos \& Depew (2004). 